\title{
ON-CHIP EDDY CURRENT SENSOR FOR CRACK DETECTION IN METALS
}

\author{
Daniel J. Sadler and Chong H. Ahn \\ Center for Microelectronic Sensors and MEMS \\ Department of Electrical and Computer Engineering and Computer Science \\ University of Cincinnati \\ P.O. Box 210030, Cincinnati, OH 45221-0030 U.S.A
}

\begin{abstract}
ABSTRACI
In this paper, we present a new integrated eddy current sensor for the detection of microcracks on the surface of metals. The device consists of two stacked planar coils fabricated onto a glass substrate and encapsulated on one side by a $\mathrm{Ni} / \mathrm{Fe}$ permalloy magnetic core. Fabrication of the device is achieved by a UVLIGA thick photoresist lithography process which involves the lithographic patterning of $15-25 \mu \mathrm{m}$ thick molds using AZ -4000 series photoresist. The introduction of the permalloy core coupled with the thick conductor lines produces a high inductance, low resistance device capable of generating large magnetic fields at low driving currents. Aluminum and titanium testing standards containing cracks 7 mils wide and 8 to 40 mils deep have been used to confirm the excellent operation of this new sensor. The device has been tested in the frequency range of $10 \mathrm{kHz}-500 \mathrm{kHz}$ and has been shown to be capable of clearly detecting cracks with depths of as little as 8 mils $(200 \mu \mathrm{m})$ at a low input power of 30 $\mathrm{mW}$. Results show an extremely linear relation between crack depth and output signal voltage with a reasonably high level of unamplified signal strength.
\end{abstract}

\section{INTRODUCTION}

Eddy current testing (ECT) is a very effective and convenient way to nondestructively evaluate a metallic surface for defects. A variety of industrial applications currently exist for nondestructive eddy current testing, especially in the aerospace industry. Although many excellent and sophisticated devices are commonly used in industry already, MFMS technology is being investigated as a way to potentially lower the cost, size, and improve the performance of these existing devices [1-4]. Recently, a variety of MEMS-based eddy current sensors have been reported for use as proximity sensors and position indicators [5-6], as well as for use in nondestructive testing for cracks and flaws in metals [1-3].

In this paper, we present a new integrated eddy current sensor for the detection of microcracks on the surface of metals. The device consists of two stacked planar coils fabricated onto a glass substrate and encapsulated on one side by a $\mathrm{Ni} / \mathrm{Fe}$ permalloy magnetic core. A UV-LIGA thick photoresist lithography process, which involves the lithographic patterning of 15-25 $\mu \mathrm{m}$ thick molds using AZ-4000 series photoresist, was used to fabricate the new eddy current sensor [7-9]. Although this new device is similar in structure and operating principle to some other previously reported devices [1-3], there are several important distinctions and improvements. First, the entire device is fabricated on one substrate with no hand-wound or attached coils. Also, the introduction of the permalloy core coupled with the thick conductor lines produces a high inductance, low resistance device capable of generating large magnetic fields at low driving currents. For these reasons, the devices presented in this work show promise for use as low cost, highly sensitive on-chip eddy current sensors.

\section{THEORY}

Two common methods exist for eddy current testing, a single coil and a dual coil method. Both rely on the principle that as a coil is excited with an $\mathrm{AC}$ voltage, flux lines emanate from one face of the coil, arch around in the space surrounding the coil, and enter the opposite face of the coil as shown in Figure 1. The total amount of flux generated is directly proportional to the amount of current flowing through the coil. The flux lines will close on themselves without crossing any other lines, but if any flux line impinges upon a conducting object, a small whirlpool like eddy current will be created at the surface of the object under test. These eddy currents, in turn produce a magnetic field which opposes that field which was generated by the driving coil. The ANSYS simulation of Figure 2 shows the change in magnetic flux linking the device that results when a conducting object is brought into the vicinity of a coil. In the single coil approach, this change in magnetic field is sensed as a change in coil inductance. The dual coil method, on the other hand, employs a second coil, called the sensing coil, which is magnetically linked to the driving coil due to its close proximity. Because the drive and sensing coils are linked, and because the generated flux is time changing (AC), an electromotive force $\left(V_{e m f}\right)$ is induced in the sensing coil as per Faraday's law which is given as follows:

$$
V_{e m f}=-N \frac{d \Psi}{d t}
$$

where $\Psi$ is magnetic flux and $N$ is the number of coil turns. Therefore, by simply monitoring the voltage coupled onto the sensing coil, changes in the magnetic field can easily be detected.

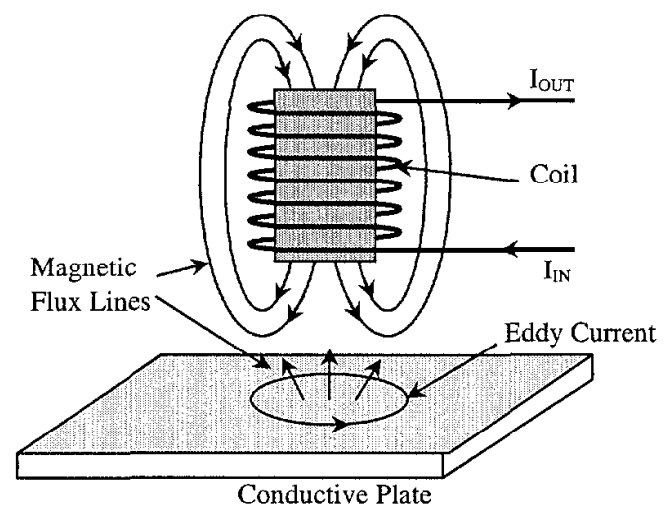

Figure 1. Magnetic fields produced by an inductor interact with the surface of a conductor to produce eddy currents. The eddy currents then produce a magnetic field which opposes the field generated by the inductor.

Although the above theoretical description is more applicable to a proximity sensor, the same analysis can be readily extended to the understanding of eddy current crack detection. Sensing the presence of the metal object can be thought of as the first order effect, while detecting defects in that object are a second order effect. Crack detection is possible by the aforementioned method due to the fact that defects in the metal object under test impede the path of eddy current flow and cause an additional change in the magnetic flux in the vicinity of the defect. Therefore, by fixing the distance between the sensor and the target, deviations in the 
measured signal can be interpreted as surface flaws in the object under test, making it possible to detect the presence of a surface flaw or crack and even characterize its dimensions.

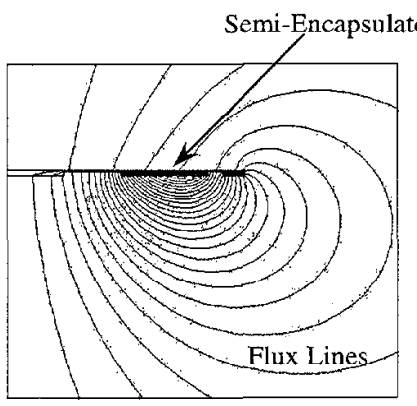

(a)

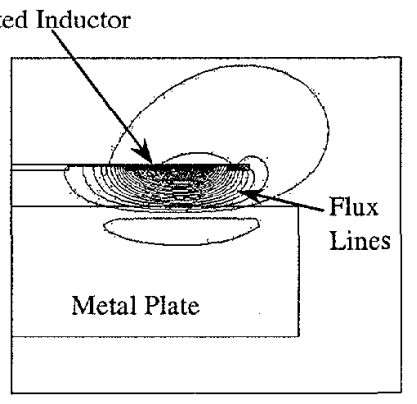

(b)
Figure 2. ANSYS simulation of flux generated by a semiencapsulated spiral inductor (a) isolated from a metal surface and (b) in the vicinity of a metal plate. Detection of this difference in flux profile (magnetic field) provides the physical basis for proximity sensors and crack detection sensors.

In this work, we have chosen the dual coil method for two reasons. First, output signals are typically larger for this method than for the single coil method [2]. Second, the actual measurement technique of simply measuring an induced voltage is more straightforward. Although this method is simple, typical problems include difficulty in device integration, low output signal strength (hundreds of microvolts or less), and high power dissipation in the driving coil. In this paper, we intend to show that through innovative design and fabrication methods, our device is able to overcome these problems and has the potential for even further improvement.

\section{DESIGN}

A schematic representation of the sensor is shown in Figure 3 . As shown in the diagram, the driving coil is located directly above the sensing coil, and the two are separated by a thin dielectric layer. In this way, the two coils are isolated electrically, but coupled magnetically due to their close proximity. For optimum performance as an eddy current sensor, coils should have both a high inductance for generating a large magnetic field and a low electrical resistance to minimize ohmic losses. In order to maximize the inductance value, a large number of turns and a tight coil packing (small conductor spacing) are required. Conversely, resistance is minimized by using conductors with a large crosssectional area and a low number of turns. In addition, device size requirements and fabrication limitations must also be taken into consideration. Because some of these requirements are contradictory, engineering tradeoffs must be made.

In the current design, both the sensing and driving coils contain 13 full turns of copper windings. Conductor line width is $50 \mu \mathrm{m}$, thickness is approximately $20 \mu \mathrm{m}$, and spacing is $30 \mu \mathrm{m}$ for both coils. This results in an overall device size of about $7 \mathrm{~mm}$ $x 7 \mathrm{~mm}$. The above dimensions were chosen in an effort to create a relatively high inductance and relatively low resistance device without unnecessarily complicating fabrication. All dimensions are easily achievable using our UV-LIGA process [7-9], and we believe that by further optimizing dimensions, a device with similar or better electrical characteristics and much smaller size can be produced with little additional fabrication effort. In addition, this technology could be extended to the development of an array-type sensor as pictured in Figure 4 which would likely be required in an actual industrial application.

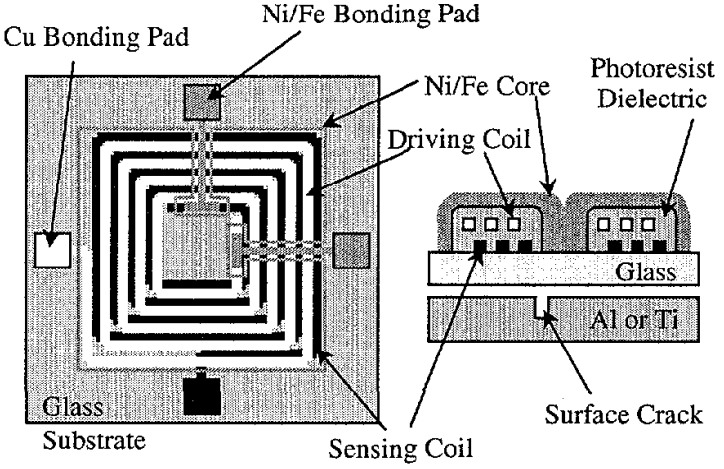

Figure 3. Top view and cross-sectional view schematic diagrams of the eddy current sensor. The entire sensor is integrated on a glass substrate including the lower sensing coil, the upper driving coil, and the semi-encapsulating magnetic core. Layers are separated by a photoresist dielectric.

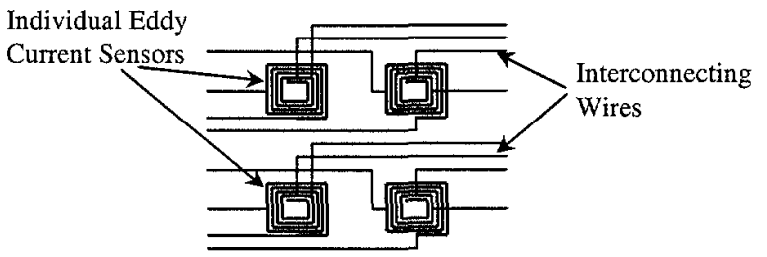

Figure 4. $2 \times 2$ array of the eddy current sensors. Individual sensors are combined to form an array for high speed scanning of a metal surface as would be required in an industrial nondestructive testing application.

\section{FABRICATION}

Brief fabrication steps for the device are shown in Figure 5. Pyrex 7740 glass wafers two inches in diameter and $250 \mu \mathrm{m}$-thick were used as substrates. Each two-inch glass substrate yielded sixteen eddy current sensors. The process began with e-beam evaporation of a Ti $(300 \AA) / \mathrm{Cu}(3000 \AA)$ seed layer. AZ-4000 series photoresist was then used to produce a $20 \mu \mathrm{m}$ thick electroplating mold. Next, copper lines were electroplated to the top of the mold using standard electroplating techniques to form the sensing coil. After removing the photoresist and seed layer, a new layer of photoresist was spun, via openings were patterned, and the photoresist was hard cured at $220^{\circ} \mathrm{C}$ to form a permanent and planarizing dielectric layer. This dielectric layer electrically insulates the lower sensing coil from the driving coil to be patterned directly above it. Another seed layer was then deposited from which the driving coil was clectroplated using the same exact method as described previously. Then photoresist and seed layers were removed and another hard curing was performed to insulate the driving coil from the semi-encapsulating magnetic core. Another seed layer was deposited and AZ-4000 series photoresist was again used, this time to pattern a mold for the semiencapsulating magnetic core. The core was formed by electroplating Ni/Fe $(81 \% / 19 \%)$ permalloy to a thickness of about $20 \mu \mathrm{m}$. It should be noted that in addition to serving as a magnetic material, this $\mathrm{Ni} / \mathrm{Fe}$ permalloy layer is also an electrical conductor and is used to form a bonding pad for making contact to the inside of the coil. This eliminates one mask from the fabrication process. Finally, photoresist and seed layers were removed to complete device fabrication. Upon completion of the microfabrication steps, wafers were diced and individual sensors packaged for testing. A microphotograph of one completed device is shown in Figure 6. 


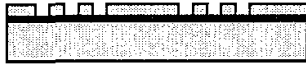

(a)

(b)

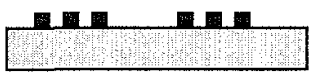

(c)

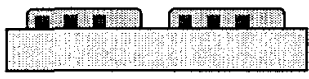

(d)

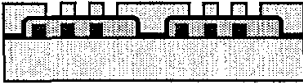

(c)

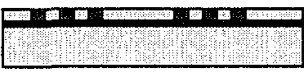

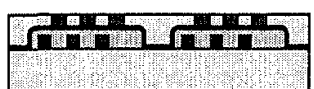

(f)

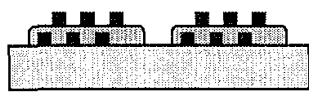

(g)

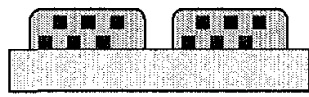

(h)

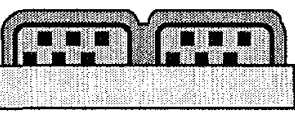

(i)
Figure 5. Brief fabrication steps: (a) Photolithography for sensing coil; (b) Copper electroplating; (c) Resist and seed layer removal; (d) High temperature curing; (e) Photolithography for driving coil; $(f)$ Copper electroplating; $(g)$ Resist and seed layer removal; (h) High temperature curing; (i) Seed layer evaporation and top magnetic core electroplating.

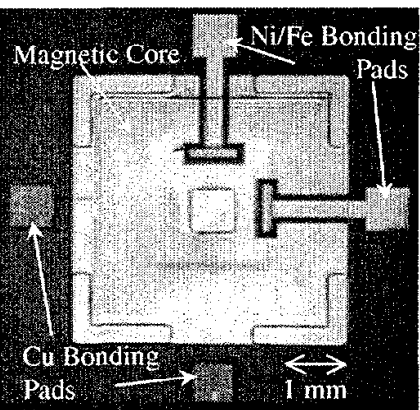

(a)

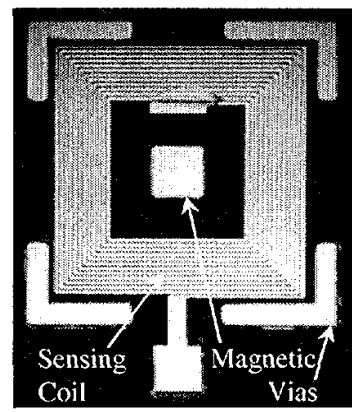

(b)
Figure 6. (a) Top view, and (b) Rear view looking through the substrate of the eddy current sensor fabricated on a Pyrex glass wafer using dual-stacked spiral inductors. Copper and Ni/Fe pads allow for connection to each of the inductors. In (a), the driving and sensing coils are hidden beneath the semi-encapsulating $\mathrm{Ni} / \mathrm{Fe}$ permalloy core. Overall sensor size is about $7 \mathrm{~mm} \times 7 \mathrm{~mm}$.

\section{RESULTS AND DISCUSSION}

Inductance and resistance measurements were performed on both the driving and sensing coils of the eddy current sensors by using a Hewlett Packard precision LCR meter. Because thickness and geometry of sensing and driving coils are the same, they have nominally the same resistance and inductance values. Figure 7 shows a graph of average inductance and resistance as a function of frequency for several sensors. The relatively low resistance ( 3 ohms) and high inductance $(1.6 \mu \mathrm{H})$ can be attributed respectively to the thick conductor lines and the semi-encapsulating $\mathrm{Ni} / \mathrm{Fe}$ permalloy core. This combination results in the generation of large magnetic fields with low power dissipation from ohmic losses.

After verifying the excellent electrical properties of the eddy current sensors, these devices were then tested for their ability to detect surface cracks in metals. Aluminum and titanium testing standards were chosen for the device characterization because these materials are used extensively in aerospace applications. The testing standards, pictured along with a sensor in Figure 8, contain cracks 7 mils wide and 8 to 40 mils deep. Devices were tested in the frequency range of $10 \mathrm{kHz}-500 \mathrm{kHz}$ and were shown to be capable of clearly detecting cracks with depths of as little as 8 mils $(200 \mu \mathrm{m})$ at a low input power of $30 \mathrm{~mW}$. Figures 9 and 10 show the device response as a function of input signal frequency when used to detect cracks of various sizes in both aluminum and titanium. In these figures, output signal refers to the RMS-voltage induced on the sensing coil when the sensor is placed over top of a crack minus the RMS-voltage induced in the sensing coil when the sensor is placed over a flawless metal surface. Overall, these results show a reasonably high level of unamplified signal strength at low input power. Additionally, testing results at a frequency of $200 \mathrm{kHz}$ are shown in Figure 11. These results show an extremely linear relationship between crack depth and output signal voltage. The excellent results achieved from these tests show that these devices have much promise for use as low cost, highly sensitive eddy current sensor arrays.

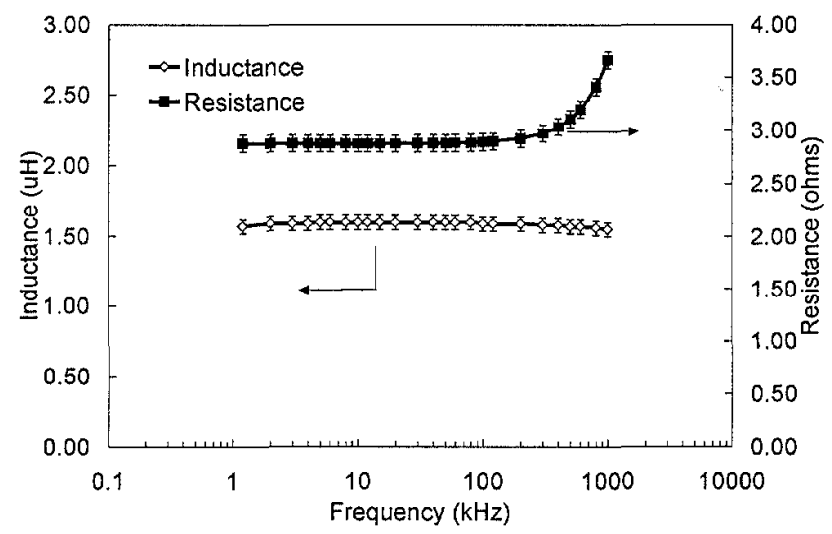

Figure 7. Inductance and resistance values as a function of frequency for spiral coils.

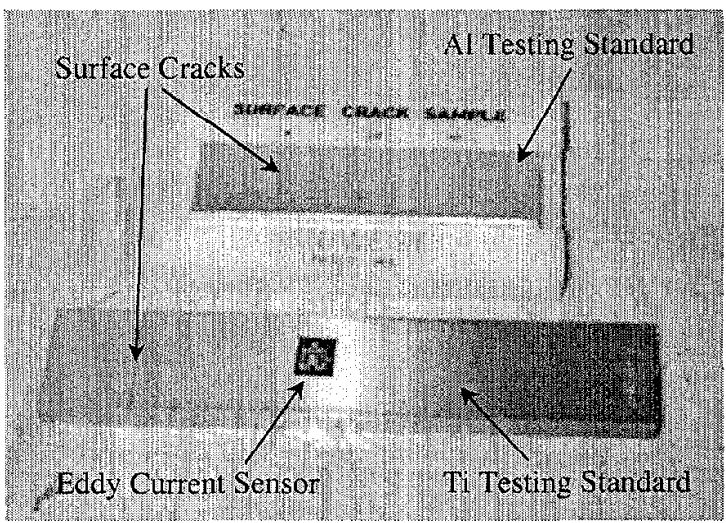

Figure 8. Aluminum and titanium testing standards pictured along with and eddy current sensor. The aluminum testing standard has three cracks with depths of 8,20 , and 40 mils, while the titanium standard has four cracks with depths of 10,20,30, and 40 mils.

\section{CONCLUSION}

In this work, a new integrated eddy current sensor for the detection of microcracks on the surface of metals has been designed, fabricated, and tested. This new sensor consists of two stacked planar coils fabricated onto a glass substrate and encapsulated on one side by a $\mathrm{Ni} / \mathrm{Fe}$ permalloy magnetic core. The sensor is fabricated by a UV-LIGA thick photoresist lithography process and is fully integrated in that it contains no hand-wound or attached coils. This UV-LIGA method allows for the formation of 
thick conductor lines, thus resulting in a low resistance device. These thick conductors, coupled with the addition of the semiencapsulating $\mathrm{Ni} / \mathrm{Fe}$ Permalloy core, produce a high inductance, low resistance device capable of generating large magnetic fields at low driving currents. Current overall device size is $7 \mathrm{~mm} \times 7$ $\mathrm{mm}$, however, by further optimizing dimensions, a sensor with similar or better electrical characteristics and much smaller size should be possible with little additional fabrication effort.

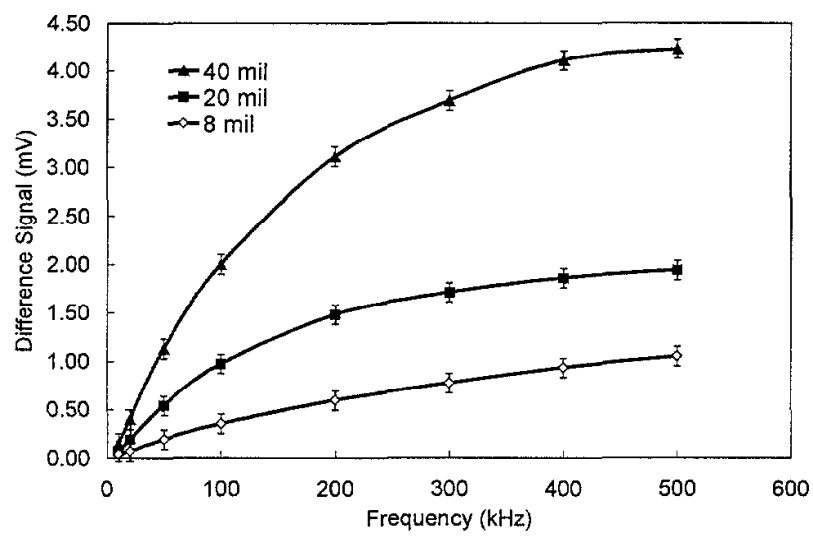

Figure 9. Difference signal vs. frequency for cracks of various depths in aluminum. Input signal power is $30 \mathrm{~mW}$.

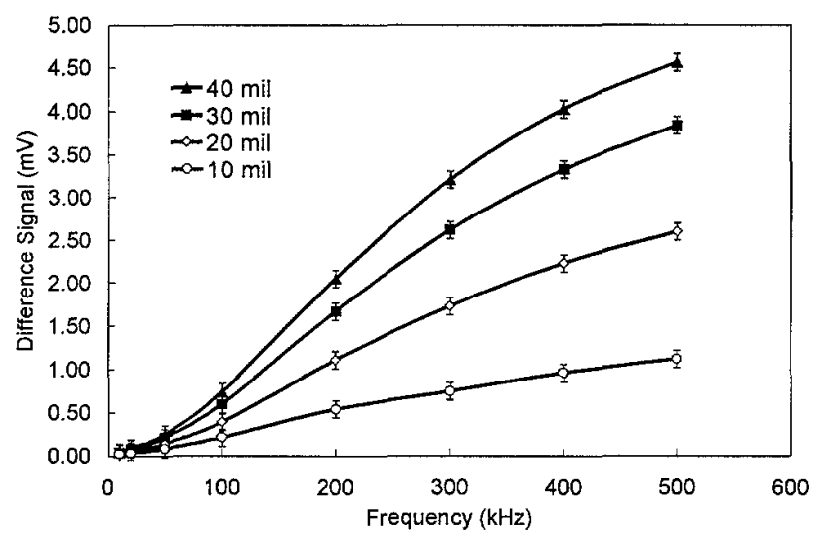

Figure 10. Difference signal vs. frequency for cracks of various depths in titanium. Input signal power is $30 \mathrm{~mW}$.

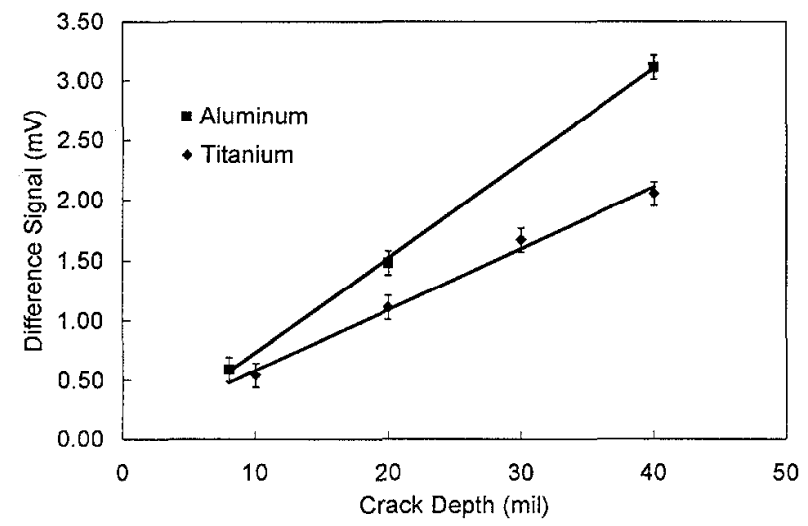

Figure 11. Output signal as a function of crack depth in both aluminum and titanium at an input frequency of $200 \mathrm{kHz}$ and input power of $30 \mathrm{~mW}$.
The sensors have been tested for their ability to detect surface cracks using both aluminum and titanium testing standards which contain cracks 7 mils wide and 8 to 40 mils deep. Tests on these samples has confirmed that these new sensors are capable of clearly detecting cracks with depths of as little as 8 mils $(200 \mu \mathrm{m})$ at a low input power of $30 \mathrm{~mW}$. Results show an extremely linear relation between crack depth and output signal voltage with a reasonably high level of unamplified signal strength. These initial results are very promising and show that these devices have great potential for use as low-cost, highly sensitive eddy current sensor arrays.

\section{ACKNOWLEDGEMENT}

The authors would like to thank the Clariant Corporation for their very generous donations of photoresist. Also, we would like to thank Mark Albers and Umang Desai from the University of Cincinnati for vital contributions to this research.

\section{REFERENCES}

1. M. Uesaka, T. Nakanishi, K. Miya, H. Komatsu, K. Aoki, and K. Kasai, "Micro Eddy Current Testing by Micro Magnetic Sensor Array", IEEE Transactions on Magnetics, Vol. 34 No. 4, 1998, pp. 2287-2297.

2. S. Yamada, M. Katou, M. Iwahara, and F. P. Dawson, "Eddy Current Testing Probe Composed of Planar Coils", IEEE Transactions on Magnetics, Vol. 31 No. 6, 1995, pp. 3185-3187.

3. Y. Hamasaki, and T. Ide, "Fabrication of Multi-Layer Eddy Current Micro Sensors for Non-Destructive Inspection of Small Diameter Pipes", IEEE Microelectromechanical Systems Workshop, Amsterdam, The Netherlands, 1995, pp. 232-236.

4. W. J. Ballantyne, "Mechatronic Applications of Eddy Current Sensing", SENSORS, January 1995, pp. 48-52.

5. P. A. Passeraub, P. A. Besse, A. Bayadroun, S. Hediger, E. Bernasconi, and R. S. Popovich, "First Integrated Inductive Proximity Sensor with On-Chip CMOS Readout Circuit and Electrodeposited $1 \mathrm{~mm}$ Flat Coil", Sensors and Actuators, A 76, 1999, pp. 273-278.

6. G. Y. Tian, Z. X. Zhao, and R. W. Baines, "The Research of Inhomogeneity in Eddy Current Sensors", Sensors and Actuators, A 69, 1998, pp. 148-151.

7. D. J. Sadler, W. Zhang, C. H. Ahn, H. J. Kim, and S. H. Han, "Micromachined Semi-Encapsulated Spiral Inductors for MEMS Applications", IEEE Trans. on Magnetics, Vol. 33 No. 5, 1997, pp. 3319-3321.

8. D. J. Sadler, T. M. Liakopoulos, and C. H. Ahn, "A New Micromachined Electromagnet with Electroplated Ni/Fe Permalloy Through-Holes," Proc. Electrochemical Society: The $5^{\text {th }}$ International Symposium on Magnetic Materials, Processes, and Devices, Boston, MA, 1998, pp. 377-388.

9. T. M. Liakopoulos, M. Xu, and C. H. Ahn, "A Micro Fluxgate Magnetic Sensor Using Micromachined 3-Dimensional Planar Coils", Proc. Solid-State Sensor and Actuator Workshop, Hilton Head, SC, 1998, pp. 19-22. 